INFORMAL REPORT

BNI -47056

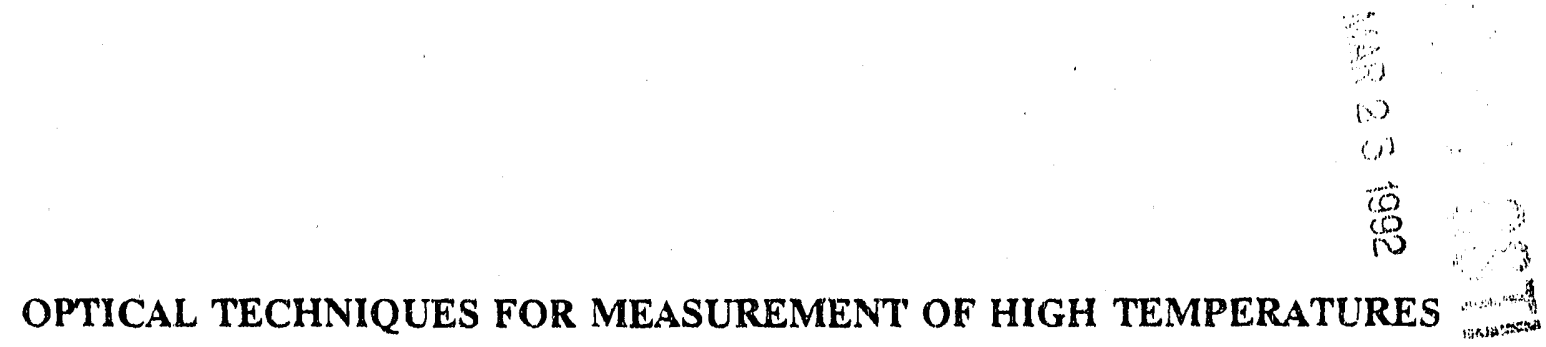

James $T$. Veligdan

October 25, 1991

Reactor Systems Division

Nuclear Energy Department

DEPARTMENT OF NUCLEAR ENERGY

BROOKHAVEN NATIONAL LABORATORY, ASSOCIATED UNIVERSITIES, INC.

UPTON, NEW YORK 11973

UNITED STATES DEPARTMENT OF ENERGY

CONTRACT NO. DE-AC02-76-CH0OO16 
This report was prepared as an account of work sponsored by an agency of the United States Government. Neither the United States Government nor any agency thereof, nor any of their employees, nor any of their contractors, subcontractors, or their employees, makes any warranty, express or implied, or assumes any legal liability or responsibility for the accuracy, completeness, or usefulness of any information, apparatus, product, or process disclosed, or represents that its use would not infringe privately owned rights. Reference herein to any specific commercial product, process, or service by traae name, trademark, manufacturer, or otherwise, does not necessarily constitute or imply its endorsement, resommendation, or favoring by the United States Government or any agency, contractor or subcontractor thereof. The views and opinions of authors expressed herein do not necessarily state or reflect those of the United States Government or any agency, contractor or subcontractor thereof. 
BNL --47056

DE92 009152

\section{OPTICAL TECHNIQUES FOR MEASUREMENT OF HIGH TEMPERATURES}

James T. Veligdan

October 25, 1991

Reactor Systems Division

Nuclear Energy Department

Brookhaven National Laboratory

Upton, Long Island, NY 11973

United States Department of Encrgy 
Table of Contents

1.0 Introduction 1

2.1) Opto-N Mechanical Expansion Thermometer 1

2.1 Sensitivity

2.2 Implementation

2.3 Accuracy

2.4 Development Time and Cost Estimate

2.5 Advantages and Disadvantages

3.0 Surface Raman Temperature Measurements 2

3.1 Sensitivity

3.2 Implementation

3.3 Accuracy and Time Response

3.4 Development Time and Cost Estimate

3.5 Advantages and Disadvantages (1)

4.) Raman Measurement of Hydrogen Temperature 3

4.1 Sensitivity 3

4.2 Implementation +

4.3 Accuracy and Time Response 4

4.4 Development Time and Cost Estimate

4.5 Advantages and Disadvantages 4

5.1) Coherent Anti-stokes Raman Spectroscopy (CARS) Thermometry of Hydrogen 5

$\begin{array}{llr}5.1 & \text { Sensitivity } & 5 \\ 5.2 & \text { Implementation } & 5 \\ 5.3 & \text { Accuracy } & 5 \\ 5.4 & \text { Development Time and Cost Estimate } & 5 \\ 5.5 \text { Advantages and Disadvantages } & 5\end{array}$

6.() Conclusions $\quad 0$

7.0) Refierences 
J. T, Veligdan

October 25, 1991

\subsection{Introduction}

The availability of instrumentation to measure the high outlet gas temperature of a particle bed reactor is a topic of some concern. There are a number ol possible techniques with advantages and disadvantages. In order to provide some baseline choice of instrumentation, a review has been conducted of these various technologies. This report summarizes the resuits of this review for a group of technologies loosely delined as opticul techniques (excluding optical pyrometry).

The review has concentrated on a number of questions for each technology investigated. 'These are:

a. Description of the technology

b. Anticipated sensitivity and accuracy

c. Requirements for implementation

d. Necessary development time and costs

e. Advantages and disadvantages of the technology

Each of these areas was considered for a technology and a large number of technologies were considered in a review of the literature. Based upon this review it was found that a large number of methods exist to measure temperarures in excess of $2000 \mathrm{~K}$. None of the methods found were ideal. Four methods, however, appeared to warrant further consideration: opto-mechanical expansion thermometry, surface Raman spectroscopy, gas-phase Raman spectroscopy and coherent anti-Stokes Raman spectroscopy (CARS). These techniques will be discussed further in this document.

\section{1) Opto-Mlechanical Expansion Thermometer}

One of the simpler methods is a mechanical expansion thermometer in which temperature is inlerred lrom a change in length of a solid due to thermal expansion. This device can be as simple as a $3 \mathrm{~mm}$ pellet made from rhenium or a refractory material which has been machined to have (wo surfaces a known distance apart. The distance between these two surlaces changes as the material expands or contracts with temperature, and this distance would be measured optically with a laser ranging device. The surlaces only need 10 be reflecting at the chosen laser wavelength, therefore, the system can operate in the intrared where poor surfaces are good reflectors.

Another embodiment of this technique would be to use the entire hot frit as an expansion pellet with a reflective face being machined into the far end of the frit itself. This technique has an additional advantage since the expansion distance is determined by measuring the frequency of the rellected laser beam. Since this is a frequency measurement, it is immune to the huge optical background.

\subsection{Sensitivity}

To estimate the sensitivity of such a device, let us assume the sensor is 0.1 inch long and has an expansion coefficient of $10 \times 10^{\circ}{ }^{\circ} \mathrm{C}^{4}$. For every degree rise in temperature, the sensor would expand one $\mu$ inch and, therefore, one would like an instrument capable of measuring physical expansion in this regime. Such a device is commercially available, a laser distance measurement system [Hewlot Packard (HP)]. It has an "oll. the-shell" accuracy of one $\mu$ inch. This HP 5528A laser measurement system would be capable of measuring comperature changes of $1^{\circ} \mathrm{C}$ with little development time since it is an "off-lhe-shelf" instrument.

Instrument response time in this case is inversely proportional to the thermal mass of the sensor. Thus, if the sensor were reduced to $0.17 \mathrm{l}$ inch long (reduced thermal mass) (o) further increatic the speed of the measurement, the sensitivity would now be about $10^{\circ} \mathrm{C}$. Sine frequency measurements are inherently more scnsitive than the pinch capability of the HP instrument, developmentat improvemems to the silock instrument might be expected to increase the sensitivity further. 
In order for this system to be operational, optical access into the hot area is required. A 2 to $3 \mathrm{~mm}$ aperture is required with either a direct line of sight or a mirrored line of sight. While this technique would be sensitive from cryogenic temperatures to the melting point of the sensor assuming the expansion cocflicient is known and a line of sight is available, the laser and its electronics must be shielded from any high radiation environments.

\subsection{Accuracy}

The accuracy of the system is inversely proportional to the required time response. The smaller the temperature sensor, the faster it comes to thermal equilibrium, but the less the absolute thermal expansion. An estimate of the achievable accuracy points toward $1^{\circ} \mathrm{C}$, while the time response should be similar to a thermocouple having the same thermal mass. (It must be remembered that the HP laser system must have its own internal temperature controlled to $1^{\circ} \mathrm{C}$.)

\subsection{Development Time and Cost Estimate}

The development time would be less than 1 year if one were to implement a totally "otf-the" shelf" system using a commercial HP laser system. Such a system would be usable for measuring the hot frit temperature by measuring the length of the hot frit itself, assuming one end of the frit is stationary. The cost for this device would be approximately $\$ 200 \mathrm{~K}$. If the HP device must be modified to measure two surfaces on a single expansion pellet, then the development time would be $11 / 2$ to 2 years; and a cost estimate for such a device would be $\$ 500 \mathrm{~K}$.

\subsection{Advantages and Disadvantages}

On the plus side, the system would be light, have low cost, short development time, and have accuracy of a few degrees. The down side--the effect of aerodynamic turbulence within the hot zone produce on the beam is unknown. A test must be performed. Further, this technique requires optical access to the hot zone and will have a time response limited by the mass of the expansion pellet.

\section{0) Surface Raman Temperature Measurements}

High temperature thermometry has been demonstrated using Raman spectra of the hexagonal phase of boron nitride (REFERENCE NEEDED). The hexagonal phase of boron nitride has the same structure as traphite; parallel planes of edge shared hexagons. Two Raman active modes corresponding 10 interplanar stretching and interplanar shear have been observed. As the cemperature of boron nitride increases. the individual B-N bonds lengthen and this Raman measurement specifically probes only the B-N bond. The sample temperatures are estimated from analysis of the Stokes and anti-Stokes line intensity ratios following normalization to the spectrometer response function.

\subsection{Sensitivity}

The temperature dependence trom ambient to $2325 \mathrm{~K}$ showed a change of $80 \mathrm{~cm}^{-1}$ in the modc irequency. This corresponds to a sensitivity of $0.04 \mathrm{~cm}^{-1} /{ }^{\circ} \mathrm{K}$. The corrected instrumental resolutions were calculated to be $0.05 \mathrm{~cm}^{-1}$. Therefore, the best obtainable accuracy is plus or minus $15^{\circ} \mathrm{K}$. 


\subsection{Implementation}

To operate a system like this, one must have a frequency doubled Nd-YAG laser and spectrometer. Such a laser weighs approximately 100 pounds and occupies about $1 / 2$ cubic meter of volume while requiring auxiliary thermal cooling. The tandem triple spectrometer occupies about the same volume while having only half the weight. The laser requires a line of sight to the hot surface to be measured. The spectrometcr also requires a line of sight (they could both use the same aperture) to the hot surface with a clear aperture of a couple $\mathrm{cm}^{2}$. The detector within the spectrometer must be shielded from ionizing radiation.

A second requirement as with the opto-mechanical thermometer is for a sensing surface. This surface must have a Raman active mode with a temperature dependent of sufficient magnitude 10 obtain the required sensitivity.

\subsection{Accuracy and Time Response}

The thermal accuracy of this system should be approximately plus or minus $15^{\circ} \mathrm{K}$ with an update time of 33 milliseconds between data acquisition points. The thermal accuracy is limited by the spectrometer resolution which in turn is limited by slit width. Therefore, for higher thermal accuracy, one would want to have narrow slits on the spectrometer and take data for a longer period of time. The use of a multichannel analyzer (OMA) although more expensive, could further reduce the data acquisition time.

\subsection{Development Time and Cost Estimate}

Since boron nitride is only usable to $2500^{\circ} \mathrm{K}$ at temperatures above this it decomposes. Further it is not incompatible with refractory carbides at the temperature of interest. Thus. another sensor must be sought. This would require a development program. Stoichiometris refractory carbide such as $T_{\Lambda} \mathrm{C}$ and $\mathrm{NbC}$ do not have a first order Raman spectrum. Although vacancies produce defect-induced Raman scattering, the temperature dependence of these modes would have to be studied. A further complication might be produced by change in stoichiometry of temperature. A pretiminary estimate is three years and $\$ 2 \mathrm{M}$.

\subsection{A.dvantages and Disadvantages}

One major advantage of this Raman technique is that you are actually measuring the lemperature of the surlace you are interested in and not just that of a probe. Anorher good point is the accuracy of the thermal measurement. The drawbacks are that you might require two optical access ports, a large laser and spectrometer, and three years of development time.

\section{1) Raman Measurement of Hydrogen Temperature}

Raman scattering is a result of inelastic, i.e., energy exchanging, collisional processes between photons of light and individual molecules. As a molecule absorbs an incident photon, the molecule is raised to some intermediate state whereby it immediately makes a Stokes transition back down to an energy level slightly above the original energy. An anti-Stokes transition to an energy slightly below the original can occur if the molecule was excited to begin with. By monitoring either the Stokes or anti-Stokes lines, one is able to determine the gas species as well as the temperature. The cross section for Raman scaltering is proportional to the fourth power of the excitation frequency. Thus, as a ternperature monitor, UV excitation would be used to achieve high ser sitivity with low background.

\subsection{Sensitivity}

The sensitivity of a Raman-based temperature sensor is dependent upon the achievable signal (1) background ratio, Since no optical background measurements have been perlormed for our particular 
application, we must make some estimates. J. A. Shirley at United Technologies has prepared a study using a Raman system to measure the temperature in the space shuttle main engine. In his linal technical report (R83-956181F), he stated that a $6^{\circ} \mathrm{K}$ accuracy is possible in a 63 millisecond time. This data acyuisition time is limited primarily by the paucity of the Raman signal itself. In our application, the number of signal counts should be approximately two orders of magnitude higher for the following two reasons:

1. We choose to operate at a shorter wavelength, $248 \mathrm{~nm}$ vs. $488 \mathrm{~nm}$ since the scattered Raman signal is proportional to $(1 / \lambda)^{4}$.

2. Our signal will be higher because our operating pressure will be an order of magnitude higher. The intensity of our background is as yet unknown, however, black body radiation in the UV should be negligibly small.

\subsection{Implementation}

In order to implement a Raman system, an optical aperture must be provided having a minimum diameter of $2 \mathrm{~cm}$ with either a direct or mirrored line of sight. The excimer laser occupies approximately 2 cubic meters and weighs about 500 pounds. The spectrometer and OMA occupy about 1 cubic meter and weigh 200 pounds. The OMA detector will have to be shielded as will the drive electronics for the laser and spectrometer. Although most Raman experiments collect the signad at $90^{\circ}$ to the incident light to maximize signal collection, such a geometry would require two windows at orthogonal positions. Backscatter geometry will require only one access window and has some advantage in terms of signal intensity.

\subsection{Accuracy and Time Response}

Since the optical backgrounds within the hot area are as yet unknown. we can only make an extrapolation from the data of J. A. Shirley (noted previously) as to the accuracy and time response. Certainly, the blackbody emission should be negligible in the UV based on preliminary experiments. and it seems possible to take the temperature measurement in a single laser shot assuming the laser emits 250 millijoules per pulse. An "off-the-shelf" excimer laser which we are considering operates at $200 \mathrm{~Hz}$ with an energy of 4.50 millijoules per pulse. It, therefore. seems plausible that the measurement $\left(\sim 10^{\circ} \mathrm{K}\right.$ accuracy $)$ can be done in approximately 10 milliseconds which is the standard counting time of the OMA. If necessary, this counting time could be shortened.

\subsection{Development Time and Cost Estimate}

The time required to implement such a system would be about two and onc half man-ycars. The equipment exists for a feasibility study and a technology demonstration of the system is planned for FY 92. Once this is complete, the optical design for implementation into PIPET can begin. The capital costs for the laser and detector are in the range of $\$ 200 \mathrm{~K}$. A complete optical system can only be designed once the details of the access system are known.

\subsection{Advantages and Disadvantages}

One great advantage of this system is that it will actually measure the mixed mean outlet temperature of the device within tens of milliseconds or less, while maintaining an accuracy of $10^{\circ} \mathrm{K}$ or better. Secondly, the gas temperature at various points can be monitorec by proper arrangement ol optics. Thirdly: calibration is intrinsic since the Raman spectrum of $\mathrm{H}_{2}$ can be directly calculated. This means that this technique can be used to calibrate other techniques.

A disadvantage of the Raman system is its size and power requirements, about 3 cubic meters and 380 volts three phase power. Another possible disadvantage is the reduction in signal due 10 hydrogen ionization. 


\section{5.) CARS Thermometry of Hydrogen}

CARS is an optical wave mixing process where two incident laser beams have a frequency difference which is appropriate to the molecule being probed. The two frequencies $w 1$ and w2 interact to generate the CARS signal $w 3=2 w 1-w 2$. The two laser frequencies are adjusted such that $w 1-w 2=w_{v 11}$ where $w_{v 10}$ corresponds to a Raman active vibrational-rotational transition in the molecule of interest. Therefore, because of the quantum mechanical resonance in such a scheme, the CARS signal is orders of magnitude stronger than conventional spontaneous Raman scattering. More importantly the CARS generated signal appears as a laserlike beam which allows almost all of the signal to be captured. Temperature determination from CARS data usually requires that the spectra be fitted to computer generated spectra. However, because of the large vibration-rotation interaction in hydrogen, the temperature might be found by observing the relative peak heights of the CARS spectra.

\subsection{Sensitivity}

Hydrogen has a simple CARS spectrum and the lines are spread out over 200 wavenumbers. The intensity contrast between adjacent lines is quite large and is, therefore. used to determine the temperature. The CARS technique has demonstrated a sensitivity of $10^{\circ} \mathrm{K}$ when operating in the $2,000^{\circ}$ range (Shirley, Eckbreth and Hall, 1979).

\subsection{Implementation}

The CARS technique is a non-linear optical process and as such requires high laser intensities to operate. Since it is also a resonant process, the laser wavelengths are precisely predetermined, unlike Raman scattering which can use a continuum of wavelengths. Because of the need for precise wavelengths. dye lasers with corresponding dye ampliliers are used. Another laser is also required which is usually a frequency doubled $\mathrm{NCl}$-YAG laser. In order to obtain a good CARS signal, the incident laser beams must all be aligned so that the wave mixing process is properly phase matched. Once the CARS signal is generated it must then be separated from the incident laser beams before it is collected. The polarization orientation of the pump lasers must be accurately controlled as well as the spatial focusing and crossing parameters. Adjustable telescopes are used to position the laser beams for proper waist crossing. The CARS scheme would require (wo optical ports. The lirst of which should be approximately $310+\mathrm{cm}$ in diameter, while the exit port need only $\mathrm{be} 2 \mathrm{~cm}$ in diamucer. The three lasers and spectrometer would require at least 4 cubic meters of space. Although the CARS lechniclue is species specilic and it has good resolution, it is not trivial to implement.

\subsection{Accuracy}

The CARS technique has already demonstrated an accuracy of $10^{\circ} \mathrm{K}$ or beller.

\subsection{Development Time and Cost Estimate}

Implementing a CARS diagnostic sensor would require approximatcly $\$ 3 M$ and $21 / 2$ ycars.

\subsection{Advantages and Disadvantages}

CARS has an advantage of actually measuring the gas temperature while being unintluenced by the surroundings. The accuracy is also quite acceptable at $110^{\circ} \mathrm{K}$. CARS has a distinct disidvantage in that it is quile difficult to implement requiring three lasers which must produce high power pulsed huamss which must be properly phased and locused. 


\section{Conclusions}

Since any research program has finite linancial resources, a down selection of temperature sensing techniques must be performed. The CARS system is simply too difficult and too complex to implement in a reasonable time. The surface Raman system has a major unknown in it at this point which is the spectrum of the sisface material itself. For this reason, the surface Raman technique is not being pursued at the present time. The optomechanical temperature probe looks promising after some initial laboratory tests. The laser must be modified to have a higher intensily, and the beam size might be expanded as tests are done to determine the ulfects of heat and turbulence on the reflected beam. Therefore, work on this technique should proceed at least to the point of an experiment in a blow-down system. During FY '92 the major emphasis of experimental work, however, will be directed toward the development of a Raman system for implementation in PIPET. Although such a system is only useful in ground tests, it can provide reliable and accurate temperature data which can be used as a calibration source. Furthermore, Raman spectroscopy does not pose any major unknowns since it is a well-established spectroscopic technique.

\subsection{References}

REIFERENCE FROM SECTION 3.0 SURFACE RAMAN TEMPERATURE MEASUREMENTS

COMPLETE REFERENCE FROM SECTION 5.1 SENSITIVITY - Shirley, Eckbreth, and Hall, 1979 

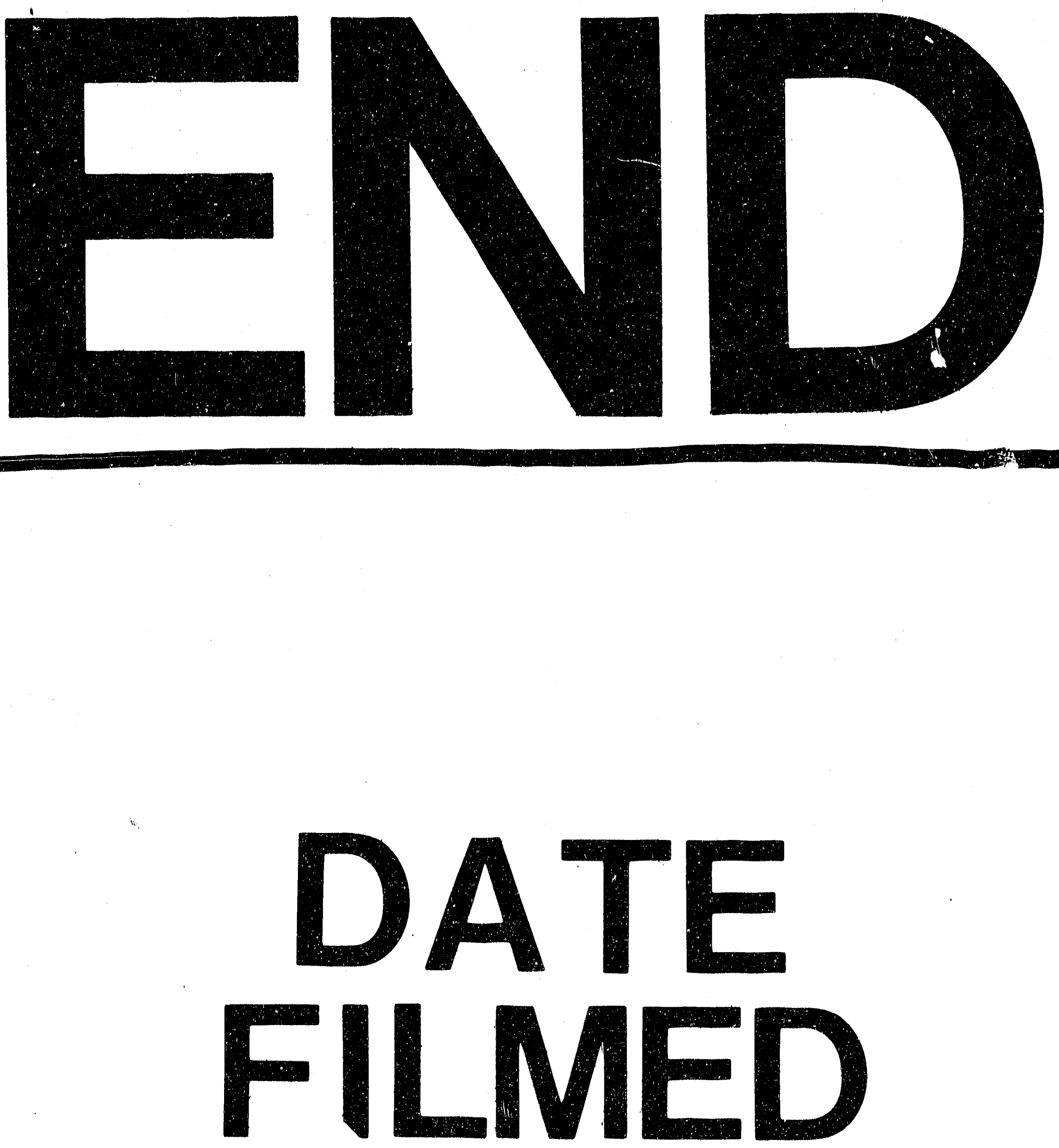

$=$
$=$
$\vdots$
$\overline{\mathbf{1}}$
$\overline{3}$
$\overline{3}$

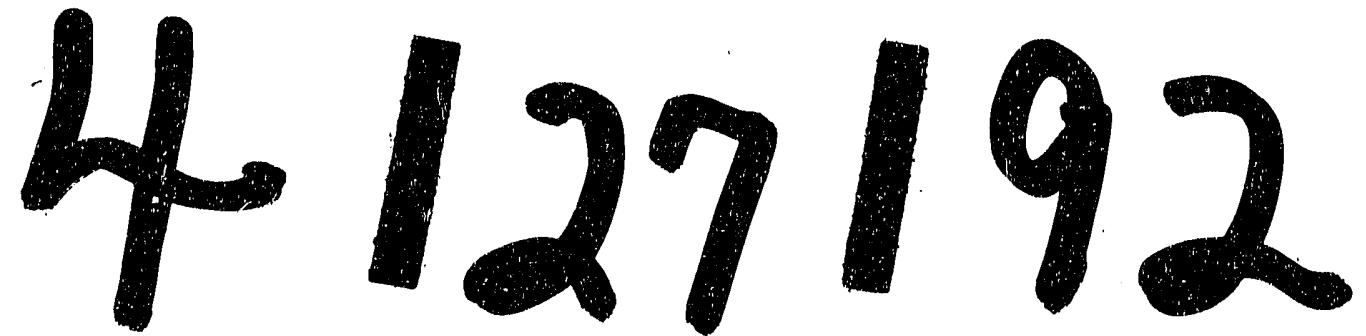


